Research Article

\title{
Relative Gottlieb Groups of Embeddings between Complex Grassmannians
}

\author{
J. B. Gatsinzi \\ Department of Mathematics and Statistical Sciences, Botswana International University of Science and Technology, \\ Palapye, Botswana
}

Correspondence should be addressed to J. B. Gatsinzi; jeangatsinzi@yahoo.fr

Received 15 October 2021; Accepted 27 October 2021; Published 15 November 2021

Academic Editor: Luca Vitagliano

Copyright $\odot 2021$ J. B. Gatsinzi. This is an open access article distributed under the Creative Commons Attribution License, which permits unrestricted use, distribution, and reproduction in any medium, provided the original work is properly cited.

Let $\operatorname{Gr}(k, n)$ be the complex Grassmann manifold of $k$-linear subspaces in $\mathbb{C}^{n}$. We compute rational relative Gottlieb groups of the embedding $i: \operatorname{Gr}(k, n) \longrightarrow \operatorname{Gr}(k, n+r)$ and show that the $G$-sequence is exact if $r \geq k(n-k)$.

\section{Introduction}

We work in the category of spaces having the homotopy type of simply connected CW complexes of finite type. We denote by $h: X \longrightarrow X_{\mathbb{Q}}$ the rationalization of $X[1,2]$. Let $f:\left(X, x_{0}\right) \longrightarrow\left(Y, y_{0}\right)$ be a pointed continuous mapping and $\operatorname{map}(X, Y ; f)$ be the component of $f$ in the space of all continuous maps $g: X \longrightarrow Y$. Consider the evaluation map ev: $\operatorname{map}(X, Y ; f) \longrightarrow Y$ at the base point $x_{0}$, that is, $\operatorname{ev}(g)=g\left(x_{0}\right)$. The $n$th evaluation subgroup of $f$, $G_{n}(Y, X ; f)$, is the image of $\pi_{n}(\mathrm{ev})$ in $\pi_{n}(Y)$ [3]. In the special case where $X=Y$ and $f=1_{X}$, one obtains the Gottlieb group $G_{n}(X)$ of $X$ [4]. Gottlieb groups play an important role in topology. For instance, if $G_{n}(X)=0$, then any fibration $X \longrightarrow E \longrightarrow S^{n+1}$ admits a section (Corollary $2-7$ in [4]).

In [2], Lee and Woo introduce relative evaluation groups $G_{n}^{\text {rel }}(Y, X ; f)$ and obtain a long sequence,

$$
\begin{aligned}
\cdots & \longrightarrow G_{n+1}^{\mathrm{rel}}(Y, X ; f) \longrightarrow G_{n}(X) \longrightarrow G_{n}(Y, X ; f) \\
& \longrightarrow G_{n}^{\text {rel }}(Y, X ; f) \longrightarrow \cdots,
\end{aligned}
$$

called G-sequence [5]. This sequence is exact in some cases, for instance, if $f$ is a homotopy monomorphism [6].

\section{Rational Relative Gottlieb Groups}

The rationalization $h: Y \longrightarrow Y_{\mathbb{Q}}$ induces a rationalization $h_{*}: \operatorname{map}(X, Y ; f) \longrightarrow \operatorname{map}(X, Y ; h \circ f)$ [7]. Therefore,

$$
\mathrm{ev}_{*}\left(\pi_{*}(\operatorname{map}(X, Y ; f)) \otimes \mathbb{Q}\right) \cong \mathrm{ev}_{*}\left(\pi_{*}\left(\operatorname{map}\left(X, Y_{\mathbb{Q}} ; h \circ f\right)\right)\right) \text {. }
$$

In this paper, we study the $G$-sequence of the natural inclusion $\mathrm{Gr}(k, n) \longrightarrow \mathrm{Gr}(k, n+r)$ using models of function spaces in rational homotopy $[8,9]$. In particular, we show that the $G$-sequence is exact if $r \geq k(n-k)$. We work with algebraic models in rational homotopy theory introduced by Sullivan and Quillen $[10,11]$. In this section, we give relevant definitions and fix notation. Details can be found in [1]. All vector spaces and algebras are over the field of rational numbers $\mathbb{Q}$.

Let $(A, d)$ be a cochain algebra. The degree of an homogeneous element $a \in A^{p}$ is written $|a|$. We assume that 
$(A, d)$ is 1-connected, that is, $H^{0}(A, d)=\mathbb{Q}$ and $H^{1}(A, d)=0$. The algebra $A$ is called commutative if $a b=$ $(-1)^{|| a|| b \mid} b a$ for homogeneous elements $a, b \in A$.

Definition 1. A commutative differential graded algebra (cdga, for short) $(A, d)$ is called a Sullivan algebra if $A=S\left(V^{\text {even }}\right) \otimes E\left(V^{\text {odd }}\right)$, where $V=\oplus_{k \geq 2} V^{k}$. It will be denoted by $(\wedge V, d)$.

Moreover, a Sullivan algebra $(\wedge V, d)$ is called minimal if $\mathrm{d} V \subset \wedge^{\geq 2} V$. A Sullivan model of $(A, d)$ is given by a Sullivan algebra $(\wedge V, d)$ together with a quasi-isomorphism $f:(\wedge V, d) \longrightarrow(A, d)$. It is unique up to isomorphism.

Definition 2. If $X$ is a simply connected space of finite type, then the (minimal) Sullivan model of $X$ is the (minimal) Sullivan model of cdga $A_{\mathrm{PL}}(X)$ of polynomial differential forms on $X[1,5]$. A simply connected topological space $X$ is called formal if there exists a quasi-isomorphism $(\wedge V, d) \longrightarrow H^{*}(X, \mathbb{Q})$, where $(\wedge V, d)$ is a Sullivan model of $X$. Formal spaces include homogeneous spaces $G / H$, where $G$ and $H$ have the same rank.

The complex Grassmann manifold $\operatorname{Gr}(k, n)$ is the space of $k$-dimensional subspaces of $\mathbb{C}^{n}$. Moreover, $G(k, n) \simeq U(n) /(U(k) \times U(n-k))$, where $U(n)$ is the unitary group. Hence, $G(k, n)$ is formal (see also [11, 12]). As $G(k, n) \cong G(n-k, n)$, we will assume that $k \leq n / 2$. As $G(k, n)$ is a formal, its Sullivan model can be computed from its cohomology algebra. Precisely,

$$
H^{*}(\operatorname{Gr}(k, n))=\frac{\wedge\left(x_{2}, x_{4}, \ldots, x_{2 k}\right)}{\left(h_{n-k+1}, \ldots, h_{n-1}, h_{n}\right)},
$$

where $h_{j}$ is the polynomial of degree $2 j$ in the Taylor expansion of the expression $1 /\left(1+x_{2}+\cdots+x_{2 k}\right)$ [13]. A Sullivan model is given by

$$
\left(\wedge\left(x_{2}, \ldots, x_{2 k}, x_{2 n-2 k+1}, \ldots, x_{2 n-1}\right), d\right),
$$

where $\mathrm{d} x_{2 i}=0$ and $\mathrm{d} x_{2 n-2 k+2 i-1}=h_{n-k+i}, \quad i=1, \ldots, k$. Moreover, this model is minimal.

Let

$$
\begin{aligned}
(\wedge V, d) & =\left(\wedge\left(x_{2}, \ldots, x_{2 k}, x_{2 n+2 r-2 k+1}, \ldots, x_{2 n+2 r-1}\right), d\right), \\
(\wedge W, d) & =\left(\wedge\left(y_{2}, \ldots, y_{2 k}, y_{2 n-2 k+1}, \ldots, y_{2 n-1}\right), d\right),
\end{aligned}
$$

be respective minimal Sullivan models of $\operatorname{Gr}(k, n+r)$ and $\operatorname{Gr}(k, n)$. A Sullivan model of the inclusion $i: \operatorname{Gr}(k, n) \longrightarrow \operatorname{Gr}(k, n+r)$ is then

$$
\phi:(\wedge V, d) \longrightarrow(\wedge W, d),
$$

which is defined by

$$
\begin{aligned}
\phi\left(x_{2}\right) & =y_{2}, \ldots, \phi\left(x_{2 k}\right)=y_{2 k}, \\
\phi\left(x_{2 n+2 r-2 k+2 i+1}\right) & =\sum_{j=0}^{k-1} p_{i j} y_{2 n-2 k+2 j+1},
\end{aligned}
$$

where $p_{i j}$ is a polynomial of degree $2(r+i-j)$ in $y_{2}, \ldots, y_{2 k}$, for $i, j=0,1,2, \ldots, k-1$, provided that $r+i-j \geq 0$.

The polynomials $p_{i j}$ encode the relationships between $h_{i}$ 's. They can be explicitly expressed from the equality:

$$
\left(1+x_{2}+\cdots+x_{2 k}\right)\left(1+h_{1}+h_{2}+\cdots\right)=1 .
$$

For instance, for $k=2$,

$$
\begin{aligned}
& h_{1}=-x_{2}, \\
& h_{2}=x_{2}^{2}-x_{4}, \\
& h_{3}=-x_{2}^{3}+2 x_{2} x_{4}, \\
& h_{4}=x_{2}^{4}-3 x_{2}^{2} x_{4}+x_{4}^{2}, \\
& h_{5}=-x_{2} h_{4}-x_{4} h_{3}, \\
& h_{6}=\left(x_{2}^{2}-x_{4}\right) h_{4}+x_{2} x_{4} h_{3}, \\
& h_{7}=h_{4} h_{3}+\left(-x_{2}^{2} x_{4}+x_{4}^{2}\right) h_{3} .
\end{aligned}
$$

Example 1. The inclusion $\operatorname{Gr}(2,4) \longrightarrow \operatorname{Gr}(2,7)$ has a Sullivan model:

$$
\phi:\left(\wedge\left(x_{2}, x_{4}, x_{11}, x_{13}\right), d\right) \longrightarrow\left(\wedge\left(y_{2}, y_{4}, y_{5}, y_{7}\right), d\right),
$$

where

$$
\begin{aligned}
\mathrm{d} x_{2} & =\mathrm{d} x_{4}=0, \\
\mathrm{~d} x_{11} & =\left(x_{2}^{2}-x_{4}\right) h_{4}+x_{2} x_{4} h_{3}, \\
\mathrm{~d} x_{13} & =h_{4} h_{3}+\left(-x_{2}^{2} x_{4}+x_{4}^{2}\right) h_{3}, \\
\mathrm{~d} y_{2} & =\mathrm{d} y_{4}=0, \\
\mathrm{~d} y_{5} & =-y_{2}^{3}+2 y_{2} y_{4}, \\
\mathrm{~d} y_{7} & =y_{2}^{4}-3 y_{2}^{2} y_{4}+y_{4}^{2}, \\
\phi\left(x_{2}\right) & =y_{2}, \\
\phi\left(x_{4}\right) & =y_{4}, \\
\phi\left(x_{11}\right) & =y_{2} y_{4} y_{5}+\left(y_{2}^{2}-y_{4}\right) y_{7}, \\
\phi\left(x_{13}\right) & =\left(-y_{2}^{2} y_{4}+y_{4}^{2}\right) y_{5}+\left(-y_{2}^{3}+2 y_{2} y_{4}\right) y_{7} .
\end{aligned}
$$

We note that $-y_{2}^{2} y_{4}+y_{4}^{2}=d\left(y_{2} y_{5}+y_{7}\right)$; therefore,

$$
\phi\left(x_{13}\right)=d\left(y_{2} y_{5}+y_{7}\right) y_{5}+d\left(y_{5}\right) y_{7}
$$

Recall that if $\phi:\left(A, d_{A}\right) \longrightarrow\left(B, d_{B}\right)$ is a map of chain complexes; the mapping cone of $\phi$, denoted by $\operatorname{Rel}(\phi)$, is defined by

$$
\operatorname{Rel}(\phi)_{*}=\left(s A_{*-1} \oplus B_{*}, D\right),
$$

where the differential is defined by $D(s a, b)=\left(-s d_{A}(a), \phi(a)+d_{B} b\right)[9]$ or p. 46 in [14]. Define chain maps $J: B_{n} \longrightarrow \operatorname{Rel}_{n}(\phi)$ and $P: \operatorname{Rel}_{n}(\phi) \longrightarrow A_{n-1}$ by $J(b)=(0, b)$ and $P(s a, b)=a$. There is an exact sequence of chain complexes: 


$$
0 \longrightarrow B_{*} \stackrel{J}{\longrightarrow} \operatorname{Rel}_{*}(\phi) \stackrel{P}{\longrightarrow} A_{*-1} \longrightarrow 0
$$

which induces a long exact sequence:

$$
\begin{aligned}
& \longrightarrow H_{n}(B) \stackrel{H_{n}(J)}{\longrightarrow} H_{n}(\operatorname{Rel}(\phi)) \stackrel{H_{n}(P)}{\longrightarrow} H_{n-1}(A) \stackrel{H_{n-1}(\phi)}{\longrightarrow} H_{n-1}(B) \longrightarrow, \\
& G_{n}(\wedge V)=\left\{[\theta] \in H_{n}(\operatorname{Der} \wedge V, \delta): \theta(v)=1, v \in V^{n}\right\} .
\end{aligned}
$$

(see Proposition 4.3 in [14]).

Definition 3. Let $\phi:(A, d) \longrightarrow(B, d)$ be a morphism of cdga's. A $\phi$-derivation of degree $k$ is a linear mapping $\theta: A^{n} \longrightarrow B^{n-k} \quad$ such that $\theta(a b)=\theta(a) \phi(b)+(-1)^{k|a|} \phi(a) \theta(b)$. We denote by $\operatorname{Der}_{n}(A, B ; \phi)$ the vector space of $\phi$-derivations of degree $n$ and by $\operatorname{Der}(A, B ; \phi)=\oplus_{n} \operatorname{Der}_{n}(A, B ; \phi)$ the $\mathbb{Z}$-graded vector space of all $\phi$-derivations. The differential on $\operatorname{Der}(A, B ; \phi)$ is defined by $\delta \theta=\mathrm{d} \theta-(-1)^{k} \theta d$. We will restrict to derivations of positive degree; however, in degree one, we only consider those derivations which are cycles.

If $\phi: A \longrightarrow A$ is the identity mapping, we simply write $\operatorname{Der} A$ for $\operatorname{Der}\left(A, A ; 1_{A}\right)$. Moreover, if $A=\wedge V$, where $\left\{v_{1}, v_{2}, \ldots\right\}$ is a basis of $V$ and $\phi:(\wedge V, d) \longrightarrow(B, d)$ is a morphism of cdga's, we denote by $\left(v_{i}, b\right)$ the unique $\phi$-derivation $\theta$ such that $\theta\left(v_{i}\right)=b$ and zero on other elements of the basis.

Define the Gottlieb group of $(\wedge V, d)$ :
Hence, $G_{*}(\wedge V) \cong \operatorname{im} H_{*}\left(\epsilon_{*}\right)$, where $\epsilon_{*}: \operatorname{Der} \wedge V \longrightarrow \operatorname{Der}(\wedge V, \mathbb{Q} ; \epsilon)$ is the postcomposition with the augmentation map $\epsilon: \wedge V \longrightarrow \mathbb{Q}$. If $X$ is simply connected and $(\wedge V, d)$ is the minimal Sullivan model of $X$, then $G_{n}(\wedge V) \cong G_{n}\left(X_{\mathbb{Q}}\right)$, where $h: X \longrightarrow X_{\mathbb{Q}}$ is the rationalization (Propostion 29.8 in[1]).

Similarly, if $\phi:(\wedge V, d) \longrightarrow(\wedge W, d)$ is a map between Sullivan algebras, then the Gottlieb group $G_{*}(\wedge V, \wedge W ; \phi)$ is defined as $H_{*}\left(\epsilon_{*}\right)$, where $\epsilon_{*}: \operatorname{Der}(\wedge V, \wedge W ; \phi) \longrightarrow \operatorname{Der}(\wedge V, \mathbb{Q} ; \epsilon)$ is the postcomposition with $\epsilon:(\wedge W, d) \longrightarrow \mathbb{Q}$. Moreover, if $\phi$ is a Sullivan model of a map $f: X \longrightarrow Y$, where $Y$ is finite, then $G_{*}(\wedge V, \wedge W ; \phi) \cong G_{n}\left(Y_{\mathbb{Q}}, X ; h \circ f\right)$, where $h: Y \longrightarrow Y_{\mathbb{Q}}$ is the rationalization map.

Let $\phi:(\wedge V, d) \longrightarrow(\wedge W, d)$ be a Sullivan model of a map $f: X \longrightarrow Y$ between simply connected spaces. It induces a chain map $\phi^{*}: \operatorname{Der}(\wedge W) \longrightarrow \operatorname{Der}(\wedge V, \wedge W ; \phi)$ by precomposition by $\phi$. We get the following commutative diagram:

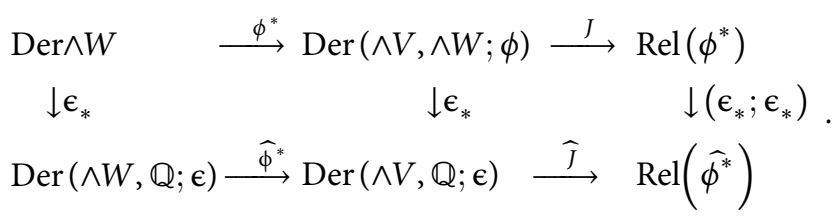

Then, rational evaluation subgroups are corresponding images in the lower ladder induced in homology by vertical maps. Therefore, there is a long sequence:

$$
\cdots \longrightarrow G_{n}(\wedge W) \stackrel{H\left(\widehat{\phi}^{*}\right)}{\longrightarrow} G_{n}(\wedge V, \wedge W ; \phi) \stackrel{H(\widehat{J})}{\longrightarrow} G_{n}^{\mathrm{rel}}(\wedge V, \wedge W ; \phi) \stackrel{H(\widehat{P})}{\longrightarrow} \cdots
$$

We will use the following result for our computations (Theorem 2.1 in [9] or Corollary 1 in [15]).

Theorem 1 (see [9]). Let $f: X \longrightarrow Y$ be a map between simply connected $C W$ complexes, where $X$ is of finite type and $\phi:(\wedge V, d) \longrightarrow(\wedge W, d)$ its Sullivan model. The long exact sequence induced by the map $f_{*}: \operatorname{map}\left(X, X ; 1_{X}\right) \longrightarrow \operatorname{map}(X, Y ; f)$ on rational homotopy groups is equivalent to the long exact sequence of

$$
\phi^{*}: \operatorname{Der}(\wedge W, d) \longrightarrow \operatorname{Der}(\wedge V, \wedge W ; \phi) .
$$

We consider the particular case, where $f$ is the inclusion $i: \operatorname{Gr}(k, n) \longrightarrow \operatorname{Gr}(k, n+r)$, where $r \geq 1$ and its Sullivan model $\phi:(\wedge V, d) \longrightarrow(\wedge W, d)$ as given in equation (6).

Theorem 2. Let $\phi:(\wedge V, d) \longrightarrow(\wedge W, d)$ be a Sullivan model of the inclusion $i: G r(k, n) \longrightarrow G r(k, n+r)$, where $r \geq k(n-k)$ :

(1) $G_{*}(\wedge V, \wedge W ; \phi) \cong V^{\#}$, the dual of $V$

(2) $G_{*}^{r e l}(\wedge V, \wedge W ; \phi) \cong s G_{*}(\wedge W) \oplus G_{*}(\wedge V, \wedge W ; \phi) \cong$ $s G_{*}(\wedge W) \oplus V^{\#}$ 
Proof

(1) Recall that $\wedge V=\wedge\left(x_{2}, \ldots, x_{2 k}, x_{2 n+2 r-2 k+1}, \ldots\right.$, $\left.x_{2 n+2 r-1}\right), \wedge W=\wedge\left(y_{2}, \ldots, y_{2 k}, y_{2 n-2 k+1}, \ldots, y_{2 n-1}\right)$, and $\phi:(\wedge V, d) \longrightarrow(\wedge W, d)$ are defined by $\phi\left(x_{2}\right)$ $=y_{2}, \ldots, \phi\left(x_{2 k}\right)=y_{2 k}, \quad \phi\left(x_{2 n+2 r-2 k+2 i-1}\right)=\sum_{j=1}^{k} p_{i j}$ $y_{2 n-2 k+2 j-1}$, and $p_{i j}$ is a polynomial of degree $2(r+$ $i-j)$ in $y_{2}, \ldots, y_{2 k}$ and $i \in\{1, \ldots, k\}$.

We consider the composition $\varphi:(\wedge V$, $d) \longrightarrow^{\phi}(\wedge W, d) \longrightarrow^{p} H^{*}(\wedge W, d)$. As $p$ is a quasiisomorphism, then the $G$-sequence of the inclusion is computed from the long exact sequence induced by the cone of the map:

$\phi^{*}: \operatorname{Der}\left(\wedge W, H^{*}(\wedge W) ; p\right) \longrightarrow \operatorname{Der}\left(\wedge V, H^{*}(\wedge W) ; \varphi\right)$.

Each of the derivations $x_{2 n+2 r-2 k+2 i+1}^{*}=$ $\left(x_{2 n+2 r-2 k+2 i-1}, 1\right) \in \operatorname{Der}\left(\wedge V, H^{*}(\wedge W) ; \varphi\right)$ is a cycle of degree at least $2 k+2 r+2 i-1>2 k+2 r$ and cannot be boundary as all even degree derivations in $\operatorname{Der}\left(\wedge V, H^{*}(\wedge W), \varphi\right)$ are of degree at most $2 k$. Hence, $\left[x_{2 n+2 r-2 k+2 i+1}^{*}\right]$ is nonzero in $G_{*}\left(\wedge V, H^{*}(\wedge W) ; \varphi\right)$

Consider the derivations $x_{2 i}^{*}=\left(x_{2 i}, 1\right) \epsilon$ $\operatorname{Der}\left(\wedge V, H^{*}(\wedge W, d), \varphi\right)$, for $i=1, \ldots, k$. Then,

$\left(\delta x_{2 i}^{*}\right)\left(x_{2 n+2 r-2 k+2 j-1}\right) \in H^{2(n+r-k+j-i)}(\wedge W, d)$.

Moreover, as $1 \leq 1, j \leq k$, then $j-i \geq-k+1$. Therefore,

$$
\begin{aligned}
2(n+r-k+j-i) & \geq 2(n+r-k-k+1) \\
& \geq 2(r+1), \quad \text { as } n \geq 2 k
\end{aligned}
$$

Therefore, $\left(\delta x_{2 i}^{*}\right)\left(x_{2 n+2 r-2 k+2 j-1}\right) \in H^{\geq 2 k(n-k)+2}=0$. Hence, $x_{2 i}^{*}$ is a cycle for $i=1, \ldots, k$. Moreover, $x_{2 i}^{*}$ cannot be a boundary as all odd degree derivations are of degree at least $2 n+2 r-2 k+1-2 k(n-k)>2(n-k)+1 \geq 2 k+1$. Therefore, $x_{2 n+2 r-2 k+2 i-1}^{*}$ are cycles which cannot be boundaries for degree reasons. Hence, $G_{*}\left(\wedge V, H^{*}(\wedge W, d), \varphi\right) \cong V^{\#}$.

(2) First, we note that $H_{\text {even }}\left(\operatorname{Der}\left(\wedge W, \wedge H^{*}(\wedge W, d) ; p\right)\right)=0$, and consequently, $G_{\text {even }}\left(\wedge W, \wedge H^{*}(\wedge W, d) ; p\right)=0 \quad[1,16]$. Moreover, a straightforward calculation shows that

$$
G_{\text {odd }}\left(\wedge W, \wedge H^{*}(\wedge W, d), p\right) \cong\left\langle y_{2 n-2 k+1}^{*}, \ldots, y_{2 n-1}^{*}\right\rangle \text {. }
$$

We consider the vector space:

$$
\operatorname{Rel}\left(\phi^{*}\right)=s \operatorname{Der}\left(\wedge W, H^{*}(\wedge W) ; p\right) \oplus \operatorname{Der}(\wedge V, \wedge W ; \varphi),
$$

where the differential is defined by $D(s \alpha, \beta)=\left(-s \delta \alpha, \phi^{*}(\alpha)+\delta \beta\right)$ Consider $W_{1}^{\#}=\left\langle y_{2 n-2 k+1}^{*}, \ldots, y_{2 n-1}^{*}\right\rangle$ in $\operatorname{Der}\left(\wedge W, H^{*}(\wedge W) ; p\right)$. For degree reasons, $\phi^{*}\left(W_{1}^{\#}\right)=0$. Therefore, $D\left(s y^{*}, 0\right)=0$, for $y^{*} \in W_{1}^{\#}$. Hence, $s y_{2 n-2 k+1}^{*}, \ldots, s y_{2 n-1}^{*}$ represent nonzero homology classes in $G_{*}^{\mathrm{rel}}\left(\wedge V, H^{*}(\wedge W) ; \varphi\right)$. We conclude that

$G_{*}^{\mathrm{rel}}\left(\wedge V, H^{*}(\wedge W) ; \varphi\right)-$

$=s G_{*}\left(\wedge W, H^{*}(\wedge W) ; p\right) \oplus G_{*}\left(\wedge V, H^{*}(\wedge W) ; \varphi\right)$.

Corollary 1. If $r \geq k(n-k)$, then the rational G-sequence of the inclusion $i: G r(k, n) \longrightarrow G r(k, n+r)$ is exact.

Proof. It comes from the previous lemma that the G-sequence is

$$
\begin{aligned}
0 \longrightarrow G_{*}\left(\wedge V, H^{*}(\wedge W) ; \varphi\right) & \longrightarrow G_{*}\left(\wedge V, H^{*}(\wedge W) ; \varphi\right) \oplus s G_{*}\left(\wedge W, H^{*}(\wedge W) ; p\right) \\
& \longrightarrow G_{*}\left(\wedge W, H^{*}(\wedge W) ; p\right) \longrightarrow 0,
\end{aligned}
$$

which is exact.

\section{Inclusion $\mathbf{G r}(k, n) \longrightarrow \mathbf{G r}(k, n+1)$}

In the range $1 \leq r<k(n-k)$, the $G$-sequence of the inclusion $\operatorname{Gr}(k, n) \longrightarrow \operatorname{Gr}(k, n+r)$ is more challenging to characterize, as shown in the following example.
Example 2. Consider the inclusion $\operatorname{Gr}(2,4) \longrightarrow \operatorname{Gr}(2,7)$ of which a Sullivan model is given by

$$
\phi: A=\left(\wedge\left(x_{2}, x_{4}, x_{11}, x_{13}\right), d\right) \longrightarrow\left(\wedge\left(y_{2}, y_{4}, y_{5}, y_{7}\right), d\right)=B
$$

where $\phi$ is defined in Example 1. We compose with the quasi-isomorphism $p:(B, d) \longrightarrow\left(H^{*}(B), 0\right)$ and consider 
$\phi^{*}: \operatorname{Der}\left(B, H^{*}(B) ; p\right) \longrightarrow \operatorname{Der}\left(A, H^{*}(B) ; \varphi\right), \quad$ where $\varphi=p \circ \phi . \quad$ Moreover, $G_{*}\left(B, H^{*}(B) ; p\right)=\left\langle\left[y_{5}^{*}\right],\left[y_{7}^{*}\right]\right\rangle$, where $y_{5}^{*}=\left(y_{5}, 1\right)$ and similarly $y_{7}^{*}=\left(y_{7}, 1\right)$. Furthermore, $\delta x_{2}^{*}=0$; hence, $\left[x_{2}^{*}\right]$ represents a nonzero homology class in $\operatorname{Der}\left(A, H^{*}(B) ; \varphi\right)$. A simple calculation shows that $\delta x_{4}^{*}=\left(x_{11}, \omega / 2\right)$, where $\omega=\left[x_{2}^{4}\right]$. Hence,

$$
G_{*}\left(A, H^{*}(B) ; \varphi\right) \cong\left\langle\left[x_{2}^{*}\right],\left[x_{11}^{*}\right],\left[x_{13}^{*}\right]\right\rangle .
$$

\section{Consider}

$\operatorname{Rel}_{*}\left(\phi^{*}\right)=\left(s \operatorname{Der}\left(B, H^{*}(B) ; p\right) \oplus \operatorname{Der}\left(A, H^{*}(B) ; \varphi\right), D\right)$.

Then,

$$
\begin{aligned}
& D\left(s y_{5}^{*}, 0\right)=\left(0, \alpha_{5}\right), \\
& D\left(s y_{7}^{*}, 0\right)=\left(0, \alpha_{7}\right),
\end{aligned}
$$

where $\alpha_{5}=\left(x_{11},\left[y_{2} y_{4}\right]\right)$ and $\alpha_{7}=\left(x_{11},\left[y_{2}^{2}-y_{4}\right]\right)$. Therefore, the image of

$$
H_{*}(P): G_{*}^{\mathrm{rel}}\left(A, H^{*}(B), \varphi\right) \longrightarrow G_{*-1}\left(B, H^{*}(B) ; p\right),
$$

is zero. Hence, the sequence

$$
G_{6}^{\mathrm{rel}}\left(A, H^{*}(B) ; \varphi\right) \stackrel{H_{*}(P)}{\longrightarrow} G_{5}\left(B, H^{*}(B) ; p\right) \stackrel{H_{5}\left(\phi^{*}\right)}{\longrightarrow} G_{5}\left(A, H^{*}(B) ; \varphi\right),
$$

reduces to

$$
0 \longrightarrow\left\langle\left[y_{5}^{*}\right]\right\rangle \longrightarrow 0
$$

$$
G_{8}^{\mathrm{rel}}\left(A, H^{*}(B) ; \varphi\right) \stackrel{H_{*}(P)}{\longrightarrow} G_{7}\left(B, H^{*}(B) ; p\right) \stackrel{H_{7}\left(\phi^{*}\right)}{\longrightarrow} G_{5}\left(A, H^{*}(B) ; \varphi\right)
$$

is not exact. Moreover, $H_{*}(J): G_{*}\left(A, H^{*}(B) ; \varphi\right) \longrightarrow G_{*}^{\text {rel }}\left(A, H^{*}(B) ; \varphi\right)$ is an isomorphism.

Although the $G$-sequence of the inclusion $\mathrm{Gr}(k, n) \longrightarrow \mathrm{Gr}(k, n+r)$ might not be exact for some values of $1 \leq r<k(n-k)$, we have the following result for $r=1$.

Theorem 3. Let $\phi:(\wedge V, d) \longrightarrow(\wedge W, d)$ be a Sullivan model of the inclusion $\mathrm{Gr}(k, n) \longrightarrow G r(k, n+1)$ :

(1) $G_{*}^{r e l}(\wedge V, \wedge W ; \phi)$ has dimension 1

(2) The G-sequence of the inclusion $G r(k, n) \longrightarrow G r(k, n+1)$ is not exact

Proof. Recall from Section 2 that the minimal Sullivan model of $\operatorname{Gr}(k, n)$ is $(\wedge W, d)$, where

$$
\begin{aligned}
W & =\left\langle y_{2}, y_{4}, \ldots, y_{2 k}, y_{2(n-k)+1}, \ldots, y_{2 n-1}\right\rangle, \\
\mathrm{d} y_{2} & =\cdots=\mathrm{d} y_{2 k}=0, \\
\mathrm{~d} y_{2(n-k+i)-1} & =h_{n-k+i}, \quad \text { for } i=1, \ldots, k .
\end{aligned}
$$

Similarly, a model of $G(k, n+1)$ is $(\wedge V, d)$, where

$$
\begin{aligned}
V & =\left\langle x_{2}, \ldots, x_{2 k}, x_{2(n-k)+3}, \ldots, x_{2 n+1}\right\rangle, \\
\mathrm{d} x_{2} & =\cdots=\mathrm{d} x_{2 k}=0, \\
\mathrm{~d} x_{2(n-k+i)+1} & =h_{n-k+i+1}, \quad \text { for } i=1, \ldots, k .
\end{aligned}
$$

which is not exact.

In the same way,
Moreover, a model of the inclusion $i: \operatorname{Gr}(k, n) \longrightarrow \operatorname{Gr}(k, n+1)$ is given by $\phi:(\wedge V, d) \longrightarrow(\wedge W, d)$ and defined by

$$
\begin{aligned}
\phi\left(x_{2 i}\right) & =y_{2 i}, \\
\phi\left(x_{2(n-k)+3}\right) & =y_{2(n-k)+3}, \ldots, \phi\left(x_{2 n-1}\right)=y_{2 n-1}, \\
\phi\left(x_{2 n+1}\right) & =-y_{2} y_{2 n-1}-y_{4} y_{2 n-3}-\cdots-y_{2 k} y_{2(n-k)+1} .
\end{aligned}
$$

We consider the quasi-isomorphism

$$
p:(\wedge W, d) \longrightarrow H^{*}(\wedge W, d)=\frac{\wedge\left(y_{2}, \ldots, y_{2 k}\right)}{\left(\mathrm{d} y_{2(n-k)+1}, \ldots, \mathrm{d} y_{2 n-1}\right)},
$$

and set $\varphi=p \circ \phi$. Consider

$$
\phi^{*}: \operatorname{Der}\left(\wedge W, H^{*}(\wedge W, d) ; p\right) \longrightarrow \operatorname{Der}\left(\wedge V, H^{*}(\wedge W) ; \varphi\right) .
$$

We have the following relations:

$$
\begin{aligned}
\phi^{*}\left(y_{2 i}^{*}\right) & =x_{2 i}^{*}, \quad \text { for } i=1, \ldots, n \\
\phi^{*}\left(y_{2 n-1}^{*}\right) & =x_{2 n-1}^{*}-\left(x_{2 n+1}, y_{2}\right) \\
\phi^{*}\left(y_{2 n-3}^{*}\right) & =x_{2 n-3}^{*}-\left(x_{2 n+1}, y_{4}\right), \\
\ldots & \\
\phi^{*}\left(y_{2(n-k)+3}^{*}\right) & =x_{2(n-k)+3}^{*}-\left(x_{2 n+1}, y_{2 k-2}\right) \\
\phi^{*}\left(y_{2(n-k)+1}^{*}\right) & =-\left(x_{2 n+1}, y_{2 k}\right) .
\end{aligned}
$$


As a result, in we have the following relations:

$\operatorname{Rel}\left(\phi^{*}\right)=s \operatorname{Der}\left(\wedge W, H^{*}(\wedge W, d) ; p\right) \oplus \operatorname{Der}\left(\wedge V, H^{*}(\wedge W) ; \varphi\right)$,

$$
\begin{aligned}
D\left(0, x_{2(n-k)+2 i+1}^{*}\right) & =0, \quad \text { for } i=1, \ldots, k \\
D\left(s y_{2 n-1}^{*}, 0\right) & =\left(0, x_{2 n-1}^{*}-\left(x_{2 n+1}, y_{2}\right)\right) \\
D\left(s y_{2 n-3}^{*}, 0\right) & =\left(0, x_{2 n-3}^{*}-\left(x_{2 n+1}, y_{4}\right)\right) \\
\ldots & \\
D\left(s y_{2 n-2 k+3}^{*}, 0\right) & =\left(0, x_{2 n-2 k+3}^{*}-\left(x_{2 n+1}, y_{2 k-2}\right)\right) \\
D\left(s y_{2 n-2 k+1}^{*}, 0\right) & =\left(0,-\left(x_{2 n+1}, y_{2 k}\right)\right) .
\end{aligned}
$$

We consider the commutative diagram:

$$
\begin{gathered}
\operatorname{Der}\left(\wedge W, H^{*}(\wedge W) ; p\right) \stackrel{\phi^{*}}{\longrightarrow} \operatorname{Der}\left(\wedge V, H^{*}(\wedge W) ; \varphi\right) \\
\downarrow \epsilon_{*} \\
\operatorname{L\epsilon } \epsilon_{*} \\
\operatorname{Der}(\wedge W, \mathbb{Q} ; \epsilon) \stackrel{\widehat{\phi}^{*}}{\longrightarrow} \operatorname{Der}(\wedge V, \mathbb{Q} ; \epsilon)
\end{gathered}
$$

Let $\widehat{y_{i}^{*}}=\epsilon_{*}\left(y_{i}^{*}\right)$ and $\widehat{x_{j}^{*}}=\epsilon_{*}\left(x_{j}^{*}\right)$. Consider

$$
\operatorname{Rel} \widehat{\phi}^{*}=(s \operatorname{Der}(\wedge W, \mathbb{Q} ; \epsilon) \oplus \operatorname{Der}(\wedge V, \mathbb{Q} ; \epsilon), \widehat{D}) \text {. }
$$

Then,

$$
\begin{aligned}
\widehat{D}\left(s \widehat{y}_{2 n-2 k+1}^{*}, 0\right) & =(0,0) \\
\widehat{D}\left(s \widehat{y}_{2 n-2 k+3}^{*}, 0\right) & =\left(0, \widehat{x}_{2 n-2 k+3}^{*}\right) \\
\ldots & \\
\widehat{D}\left(s \widehat{y}_{2 n-1}^{*}, 0\right) & =\left(0, \widehat{x}_{2 n-1}^{*}\right) .
\end{aligned}
$$

Hence,

$$
H_{*}\left(\operatorname{Rel} \widehat{\phi}^{*}\right)=\left\langle\left[\left(s \widehat{y}_{2 n-2 k+1}^{*}, 0\right)\right],\left[\left(0, \widehat{x}_{2 n+1}^{*}\right)\right]\right\rangle .
$$

Moreover, the image of $H_{*}\left(\epsilon_{*}, \epsilon_{*}\right): H_{*}\left(\operatorname{Rel} \phi^{*}\right) \longrightarrow H_{*}\left(\operatorname{Rel} \widehat{\phi}^{*}\right)$ is $\left\langle\left[\left(0, \hat{x}_{2 n+1}^{*}\right)\right]\right\rangle$. Therefore,

$$
G_{*}^{\mathrm{rel}}\left(\wedge V, H^{*}(\wedge W) ; \varphi\right)=\left\langle\left[\left(0, \widehat{x}_{2 n+1}^{*}\right)\right]\right\rangle .
$$

This shows the first part of the theorem and corrects Theorem 3 in [17] and Theorem 3 [18].

Moreover,

$H(\widehat{P}): G_{*}^{\mathrm{rel}}\left(\wedge V, H^{*}(\wedge W, d) ; \varphi\right) \longrightarrow G_{*-1}\left(\wedge W, H^{*}(\wedge W, d) ; p\right)$

is the zero map. The $G$-sequence then reduces to exact portions

$G_{2 n-2 k+2 i+1}\left(\wedge W, H^{*}(\wedge W) ; p\right) \stackrel{H\left(\widetilde{\phi}^{*}\right)}{\longrightarrow} G_{2 n-2 k+2 i+1}\left(\wedge V, H^{*}(\wedge W) ; \varphi\right)$, for $i=1, \ldots, k-1$, and

$$
G_{2 n+1}\left(\wedge V, H^{*}(\wedge W) ; p\right) \stackrel{H(\widehat{J})}{\simeq} G_{2 n+1}^{\mathrm{rel}}\left(\wedge V, H^{*}(\wedge W) ; \varphi\right),
$$

and a nonexact part,

$$
0 \longrightarrow G_{2 n-2 k+1}\left(\wedge W, H^{*}(\wedge W) ; p\right) \longrightarrow 0 .
$$

Example 3. We consider a model of the inclusion $\operatorname{Gr}(2,4) \longrightarrow \operatorname{Gr}(2,5)$ which is of the form

$$
\begin{aligned}
\phi:(\wedge V, d) & =\left(\wedge\left(x_{2}, x_{4}, x_{7}, x_{9}\right), d\right) \longrightarrow\left(\wedge\left(y_{2}, y_{4}, y_{5}, y_{7}\right), d\right) \\
& =(\wedge W, d),
\end{aligned}
$$

where $\quad \mathrm{d} x_{2}=\mathrm{d} x_{4}=0, \quad \mathrm{~d} x_{7}=x_{2}^{4}-3 x_{2}^{2} x_{4}+x_{4}^{2}$, $\mathrm{d} x_{9}=-x_{2}\left(x_{2}^{4}-3 x_{2}^{2} x_{4}+y_{4}^{2}\right)-x_{4}\left(-x_{2}^{3}+2 x_{2} x_{4}\right), \quad \mathrm{d} y_{2}=$ $\mathrm{d} y_{4}=0, \quad \mathrm{~d} y_{5}=-y_{2}^{3}+2 y_{2} y_{4}, \quad \mathrm{~d} y_{7}=y_{2}^{4}-3 y_{2}^{2} y_{4}+y_{4}^{2}$, $\phi\left(x_{2}\right)=y_{2}, \quad \phi\left(x_{4}\right)=y_{4}, \quad \phi\left(x_{7}\right)=y_{7}, \quad$ and $\phi\left(x_{9}\right)=-y_{2} y_{7}-y_{4} y_{5}$.

We compose which the quasi-isomorphism $p:(\wedge W, d) \longrightarrow H^{*}(\wedge W, d) \quad$ to get $\varphi:(\wedge V, d) \longrightarrow$ $H^{*}(\wedge W, d)$. In

$\operatorname{Rel}(\phi)_{*}=s \operatorname{Der}\left(\wedge W, H^{*}(\wedge W, d) ; p\right) \oplus \operatorname{Der}\left(\wedge V, H^{*}(\wedge W, d), \varphi\right)$,

we have the following relations: 


$$
\begin{aligned}
& D\left(\left(s y_{5}^{*}, 0\right)\right)=\left(0,\left(x_{9},-y_{4}\right)\right), \\
& D\left(\left(s y_{7}^{*}, 0\right)\right)=\left(0, x_{7}^{*}+\left(x_{9},-y_{2}\right)\right) .
\end{aligned}
$$

Consider

$$
\operatorname{Rel} \widehat{\phi}^{*}=(s \operatorname{Der}(\wedge W, \mathbb{Q} ; \epsilon) \oplus \operatorname{Der}(\wedge V, \mathbb{Q}, \epsilon), D) \cong\left(s W^{\#} \oplus V^{\#}, D\right)
$$

where

$$
\begin{aligned}
D\left(s \widehat{y}_{5}^{*}, 0\right) & =(0,0), \\
D\left(s \widehat{y}_{7}^{*}, 0\right) & =\left(0, \widehat{x}_{7}^{*}\right), \\
D\left(0, \widehat{x}_{7}^{*}\right) & =D\left(0, \widehat{x}_{9}\right)=(0,0) .
\end{aligned}
$$

Hence,

$$
H_{*}\left(\operatorname{Rel} \widehat{\phi}^{*}\right) \cong\left\langle\left[\left(s \widehat{y}_{5}^{*}, 0\right)\right],\left[\left(0, \widehat{x}_{9}^{*}\right)\right]\right\rangle .
$$

However, $\operatorname{im} H\left(\epsilon_{*}, \epsilon_{*}\right)=\left\langle\left[\left(0, \hat{x}_{9}^{*}\right)\right]\right\rangle . \quad$ Therefore, $G_{*}^{\text {rel }}\left(\wedge V, H^{*}(\wedge W, d) ; \varphi\right) \cong\left\langle\left[\left(0, \hat{x}_{9}^{*}\right)\right]\right\rangle . \quad$ As $G_{*}\left(\wedge V, H^{*}(\wedge W, d) ; \varphi\right) \cong\left\langle\left[\widehat{x}_{7}^{*}\right],\left[\widehat{x}_{9}^{*}\right]\right\rangle \quad$ and $G_{*}\left(\wedge W, H^{*}(\wedge W, d), p\right)=\left\langle\left[\hat{y}_{5}^{*}\right],\left[\hat{y}_{7}^{*}\right]\right\rangle$, then the $G$-sequence reduces to exact nonzero fragments:

$$
0 \longrightarrow G_{9}\left(\wedge V, H^{*}(\wedge W, d) ; \varphi\right) \stackrel{\cong}{\longrightarrow} G_{9}^{\text {rel }}\left(\wedge V, H^{*}(\wedge W, d) ; \varphi\right) \longrightarrow 0,0 \longrightarrow G_{7}\left(\wedge W, H^{*}(\wedge W, d) ; p\right) \stackrel{\cong}{\longrightarrow} G_{7}\left(\wedge V, H^{*}(\wedge W, d) ; \varphi\right) \longrightarrow 0,
$$

and a nonexact sequence,

$$
0 \longrightarrow G_{5}\left(\wedge W, H^{*}(\wedge W, d) ; p\right) \longrightarrow 0 .
$$

\section{Data Availability}

No data were used to support the findings of the study.

\section{Conflicts of Interest}

The author declares that there are no conflicts of interest.

\section{References}

[1] Y. Félix, S. Halperin, and J.-C. Thomas, Rational Homotopy Theory, Graduate Texts in Mathematics, Springer-Verlag, New-York, NY, USA, 2001.

[2] K. Y. Lee and M. H. Woo, "The relative evaluation sugbgroups of a CW-pair," Journal of the Korean Mathematical Society, vol. 25, pp. 149-160, 1988.

[3] J. R. Kim and M. H. Woo, "Certain subgroups of homotopy groups," Journal of the Korean Mathematical Society, vol. 21, pp. 109-120, 1984.

[4] D. H. Gottlieb, "Evaluation subgroups of homotopy groups," American Journal of Mathematics, vol. 91, no. 3, pp. 729-756, 1969.

[5] K. Y. Lee and M. H. Woo, "The G-sequence and the $\omega$-homology of a CW-pair," Topology and Its Applications, vol. 52, no. 3, pp. 221-236, 1993.

[6] J. Z. Pan and M. H. Woo, "Exactness of G-sequences and monomorphismst 2 The first author is supported by NSFC Project 19701032 and ZD 9603 of the Chinese Academy of Science, and the second author is supported by the Korea Research Foundation made in the program year of 1998 and TGRC-KOSEF 99," Topology and its Applications, vol. 109, no. 3, pp. 315-320, 2001.

[7] P. Hliton, J. Roitberg, and R. Steiner, On Free Maps and Free Homotopies into Nilpotent Spaces, Algebraic Topology, Springer, Berlin, Germany, 1977.
[8] A. Haefliger, "Rational homotopy of the space of sections of a nilpotent bundle," Transactions of the American Mathematical Society, vol. 273, no. 2, p. 609, 1982.

[9] G. Lupton and S. B. Smith, "Rationalized evaluation subgroups of a map I: Sullivan models, derivations and G-sequences," Journal of Pure and Applied Algebra, vol. 209, no. 1, pp. 159-171, 2007.

[10] D. Quillen, "Rational homotopy theory," Annals of Mathematics, vol. 90, no. 2, pp. 205-295, 1969.

[11] D. Sullivan, "Infinitesimal computations in topology," Publications Mathématiques de L'IHÉS, vol. 47, no. 1, pp. 269-331, 1977.

[12] P. Deligne, P. Griffiths, J. Morgan, and D. Sullivan, "Real homotopy theory of Kahler manifolds," Inventiones Mathematicae, vol. 29, no. 3, pp. 245-274, 1975.

[13] M. Hoffman, "Endomorphisms of the cohomology of complex Grassmannians," Transactions of the American Mathematical Society, vol. 281, no. 2, p. 745, 1984.

[14] S. Mac Lane, Homology, Springer-Verlag, Berlin, Germany, 1995.

[15] U. Buijs and A. Murillo, "The rational homotopy Lie algebra of function spaces," Commentarii Mathematici Helvetici, vol. 83, pp. 723-739, 2008.

[16] H. Shiga and M. Tezuka, "Rational fibrations homogeneous spaces with positive Euler characteristics and Jacobians," Annales de l'Institut Fourier, vol. 37, no. 1, pp. 81-106, 1987.

[17] O. Maphane, "Derivations of a Sullivan model and the rationalized G-sequence," International Journal of Mathematics and Mathematical Sciences, vol. 2021, Article ID 6687527, 5 pages, 2021.

[18] P. A. Otieno, J. B. Gatsinzi, and V. Onyango-Otieno, "Rationalized evaluation subgroups of mapping spaces between complex Grassmannians," Afrika Matematika, vol. 31, no. 2, pp. 297-303, 2020. 